\title{
L'usine hydraulique d'accumulation hebdomadaire de Montezic
}

\author{
par E. Morenon \\ Chef du Service letudes de la R.E.H. Massif Central-Pyrénées, \\ E.D.F., Toulouse
}

L'installation de transfert d'énergie par pompage de Montezic n'est à l'heure actuelle qu'au stade du projet. Des choix restent encore à faire ainsi que des études d'optimisation. La réalisation n'est pas prévue avant les années 1974-1975.

Le site de Montezic s'est révélé lors du recensement des sites de pompage qu'a fait entreprendre la Direction de l'Equipement de l'Electricité de France lorsque est apparue la nécessité prochaine du pompage.

La future installation se situera dans le département de l'Aveyron, en bordure d'un affluent du Lot: la Truyère. Cette rivière qui prend sa source dans les monts de la Margeride est déjà très équipée du point de vue hydroélectrique; pour une puissance installée, tout au long de son cours, de $740 \mathrm{MVA}$, elle produit en année moyenne près de 1500 GWh.

Au droit de la retenue créée par le barrage de Couesque, son lit est relativement encaissé et le plateau de la Viadène la domine, sur sa rive gauche, de plus de $400 \mathrm{~m}$. Sur ce plateau, dans la région de Montezic, le ruisseau de la Plane, petit affluent de la Truyère, draine une cuvette très étendue et très propice à la création d'une vaste retenue.

Le réservoir de Couesque entre sa cote de retenue normale 295,5 et sa limite inférieure d'utilisation 286,5, offre une capacité utile de $20 \mathrm{Mm}^{3}$. Au droit du site de Montezic, la capacité qu'il est possible d'utiliser (cote 280), au prix d'un arrêt passager de l'usine de Couesque, est de $30 \mathrm{Mm}^{3}$. Ce volume important dont la contrepartie pouvait être trouvée dans de bonnes conditions économiques sur le plateau a conduit à projeter une usine caractérisée par une longue durée d'utilisation journalière dans le cadre d'un cycle de fonctionnement hebdomadaire parfait.

On sait en effet que le passage du cycle journalier, au cycle hebdomadaire parfait, nécessite de multiplier la capacité de la retenue par 4,3, ou par 6 , selon que la durée d'utilisation journalière de la pleine puissance est de 10 ou $12 \mathrm{~h}$. Seuls les sites où le coût des ouvrages de stockage est bas permettent d'envisager de tels modes de fonctionnement.

La géologie qui a fortement conditionné Ia structure de l'installation se présente également très favorablement. La vallée de la Truyère, y compris ses versants, se situe dans des schistes de bonne tenue tandis que le plateau est formé par des granites extrêmement massifs et compacts dès que l'on a franchi en profondeur la zone d'altération superficielle qui, de puissance assez irrégulière, peut atteindre une trentaine de mètres. Le contact entre les schistes et les granites qui apparaît en surface en gros à la rupture de pente du versant se caractérise en profondeur par l'interpénétration sur une certaine distance de langues horizontales formées par chacun des matériaux. Aucune anomalie d'ordre géologique ne vient poser de problème particulier dans l'emprise des ouvrages projetés.

La retenue supérieure (fig. 1).

La retenue supérieure qui utilise la cuvette dont on vient de parler est créée par la construction de deux barrages:

- un barrage principal qui barre, au droit d'un étranglement, la vallée du ruisseau de la Plane;

- un barrage secondaire, de bien moindre importance, nécessité par la présence d'un ensellement

Pour obtenir la capacité souhaitée de $30 \mathrm{Mm}^{3}$, il est nécessaire d'atteindre la cote de retenue normale 703. Le plan d'eau ainsi créé aura une superficie de 260 ha.

Deux limites inférieures d'utilisation sont prévues:

- la première, fixée à la cote 692 , constitue une capacité de $20 \mathrm{Mm}^{3}$. C'est le volume que l'on peut utiliser dans la retenue inférieure sans perturber le fonctionnement de l'usine de Couesque. II permettra le fonctionnement de l'installation de Montezic, sous sa pleine puissance, pendant $10 \mathrm{~h}$ tous les jours ouvrables;

- la seconde, plus basse, fixée à la cote 681 , ne sera atteinte qu'en conjoncture exceptionnelle. Elle porte la capacité utilisable à $30 \mathrm{Mm}^{3}$ permettant à Montezic de turbiner, sous sa pleine puissance, pendant $12 \mathrm{~h}$, mais cette fois au prix d'un arrêt de l'usine de Couesque en début de semaine et jusqu'à ce que le seuil de fonctionnement soit de nouveau atteint.

Sous la même puissance installée, un fonctionnement selon le cycle journalier n'aurait nécessité qu'une capacité de $5 \mathrm{Mm}^{3}$, mais les puissances recueillies lors du fonction- 


\section{E. MORENON}

nement en turbine n'auraient été que les six et cinq dizièmes de cette puissance, selon la durée d'utilisation : 10 ou $12 \mathrm{~h}$.

L'usine aurait alors fourni de la «puissance de pointe 》 et non pas de la «puissance garantie». La valorisation apportée compense le supplément du coût qui ne concerne que le réservoir supérieur puisque le réservoir inférieur est gratuit.

Les cotes de fonctionnement des réservoirs amont et aval définies, il est possible de donner les hauteurs de chute sous lesquelles travaillera l'usine de Montezic:

Fonctionnement à :

$\left(30 \mathrm{Mm}^{3}\right)$

$\left(20 \mathrm{Mm}^{3}\right)$

Réservoir de Montezic:

$\begin{array}{lll}\text { - Cote maximale. . . . . . . . } & 703 & 703 \\ \text { - Cote minimale. . . . . . . } & 681 & 692 \\ \text { Réservoir de Couesque : } & & \\ \text { - Cote maximale. . . . . . . } & 295,5 & 295,5 \\ \text { - Cote minimale. . . . . . . . } & 280 & 286\end{array}$

Hauteurs de chutes brutes:

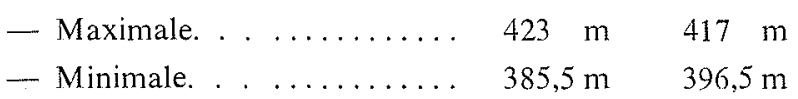

Dans les situations extrêmes, les hauteurs de chutes brutes varient de $423 \mathrm{~m}$ à $385,5 \mathrm{~m}$.

\section{Les barrages (fig. 2)}

Le profil en travers des zones à barrer ainsi que la nature des fondations ne permettent pas de concevoir des ouvrages en béton, aussi le choix s'est-il fixé sur des ouvrages en enrochements. L'absence dans le site de gisement de «terre à noyau » a fait choisir la solution avec un masque amont étanche qui sera, fort probablement, un masque en béton bitumineux sur couche drainante.

Le volume des matériaux nécessaire à la construction de ces deux barrages s'élève à $1880000 \mathrm{~m}^{3}$ :

- $1420000 \mathrm{~m}^{3}$ pour le barrage principal;

- $460000 \mathrm{~m}^{3}$ pour le barrage secondaire.

Compte tenu des volumes à mettre en place, le choix de la carrière s'avérait de première importance. Les prospections faites n'ont pas permis de découvrir dans un périmètre raisonnable une zone où l'enrochement pouvait être extrait sans conduire à la mise à la décharge d'un volume important soit de granite altéré sous forme de «gore», soit de granite ayant déjà amorcé un processus d'altération. Dans ces conditions, la meilleure solution a été de prévoir la carrière tout près de la rive gauche du barrage principal, sur le tracé des ouvrages d'adduction haute pression et de concevoir un barrage zoné de telle sorte que tous les matériaux extraits soient utilisables. La partie amont du barrage, afin d'éviter des tassements sous le masque et d'obtenir une perméabilité parfaite est prévue réalisée par des enrochements, tandis que le «gore 》 ainsi que les matériaux plus ou moins altérés seraient mis en place dans la partie aval du barrage. Une reconnaissance par sondages de la zone choisie a permis de distinguer, in situ, les deux natures de matériaux et de déterminer, en plan, la forme de la carrière qui fournirait dans les proportions désirées les deux catégories de matériaux. Cette forme est celle d'une raquette constituant un appendice à la retenue, qui, à la différence d'un chenal d'adduction à ciel ouvert, étroit et régulier, n'a pas à être traité sur son radier et ses talus. L'excavation restera brute.

Cette solution a l'avantage:

1) De situer la carrière à proximité des ouvrages à réaliser et d'éviter ainsi des frais de transport;

2) De pouvoir donner à la prise d'eau la forme souhaitée sans difficulté alors que tout contre le barrage la topographie et la géologie n'étaient pas favorables;

3) Enfin, et, c'est le plus important, de raccourcir de $510 \mathrm{~m}$ la longueur de la canalisation haute pression. Cet avantage est non seulement d'ordre financier, mais il est important au regard des régimes transitoires.

Le rapport entre la distance horizontale $L$ séparant les réservoirs et la hauteur de chute $\mathrm{H}$ caractérise grossièrement la valeur d'un site. Ce rapport qui est de $1750 / 400=4,4$, sans le chenal créé par la carrière, devient $1240 / 400=3,2$ si l'on prend en compte celui-ci. Cette valeur est tout à fait satisfaisante.

Le barrage principal aura donc les caractéristiques suivantes:

- cote du thalweg. . . ............... 648;

- cote du couronnement. . . .......... 705;

- hauteur au-dessus du thalweg......... $57 \mathrm{~m}$;

- fruit amont. . ............... 1,8/1;

- fruit aval. . ................. 1,5/1;

- revêtement étanche en béton bitumineux sur couche drainante;

- longueur en crête................ $600 \mathrm{~m}$.

Il est prolongé sur la rive droite par une digue de $420 \mathrm{~m}$, dont la hauteur varie de 0 à $12 \mathrm{~m}$ environ.

Le barrage secondaire présentant les mêmes caractéristiques aura une hauteur de $28 \mathrm{~m}$ et une longueur en crête de $600 \mathrm{~m}$.

Signalons enfin que la création de la retenue supérieure ne pose pratiquement pas de problèmes humains. Les terres noyées par la retenue ne sont pas de grande valeur, aucun village n'est touché par les eaux, trois fermes seulement sont appelées à disparaître.

Les rétablissements de communications sont peu importants. $3,8 \mathrm{~km}$ de routes départementales sont à construire. Elles emprunteront sur $1,5 \mathrm{~km}$ le couronnement des ouvrages.

\section{La retenue inférieure.}

Nous en avons déjà parlé. Disons seulement qu'elle reçoit à l'amont la restitution de l'usine de Brommat dont Electricité de France réalise actuellement le suréquipement, le débit équipé de l'usine devant passer de $95 \mathrm{~m}^{3} / \mathrm{s}$ à $200 \mathrm{~m}^{3} / \mathrm{s}$.

La liaison entre les retenues supérieure et inférieure (fig. 3).

Plusieurs schémas de la liaison entre les retenues qui comprend l'adduction haute pression, l'usine, l'adduction basse pression ont été étudiés. Le schéma entièrement souterrain s'est avéré être le meilleur. Il s'accommode fort bien de la nécessité de maintenir sur la roue des pompes, dans le cas le plus défavorable de fonctionnement, une contrepression, ici de l'ordre de $40 \mathrm{~m}$. Ce parti étant choisi, il était très tentant d'envisager de réaliser l'adduction haute pression sans avoir à la revêtir d'un blindage métallique. 


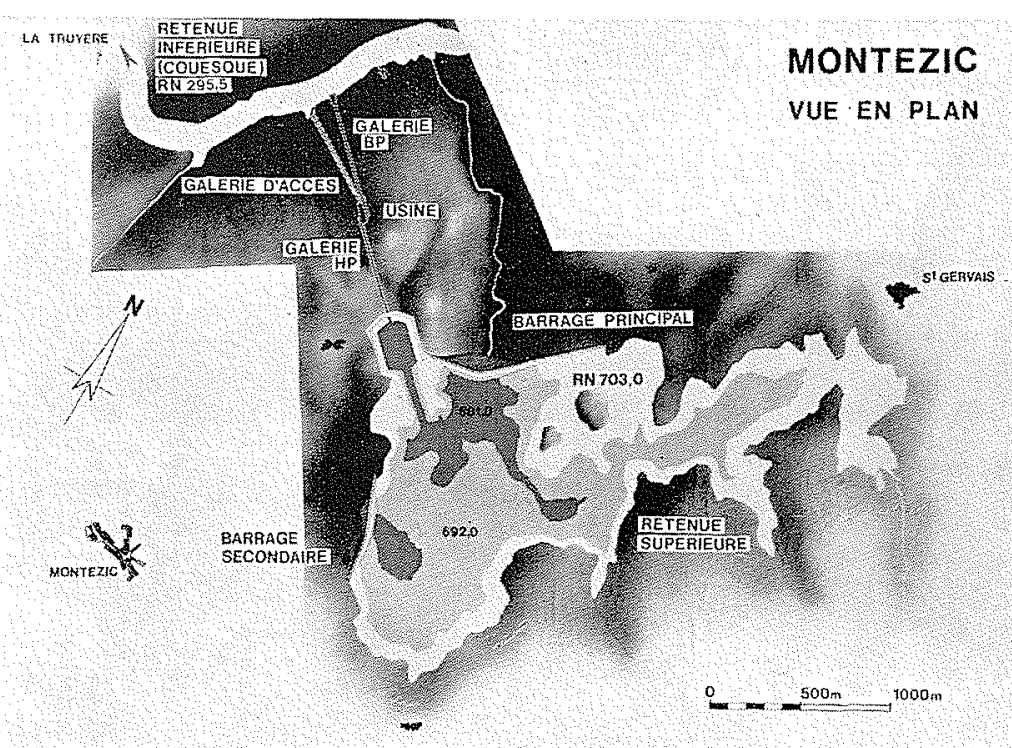

(1)

MONTEZIC

BARRAGE

COUPE TYPE
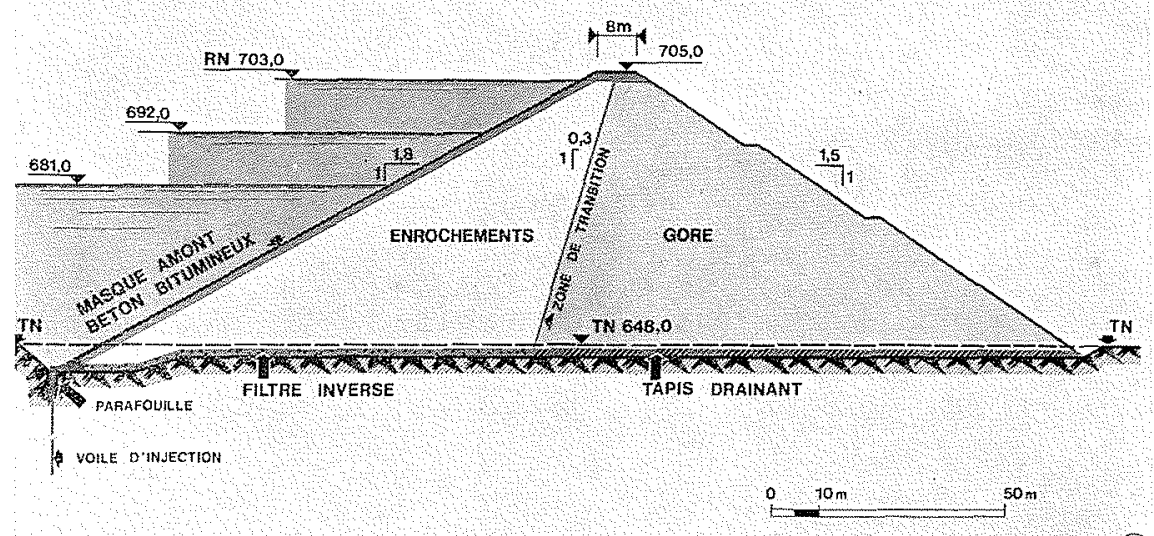

MONTEZIC

PROFIL EN LONG

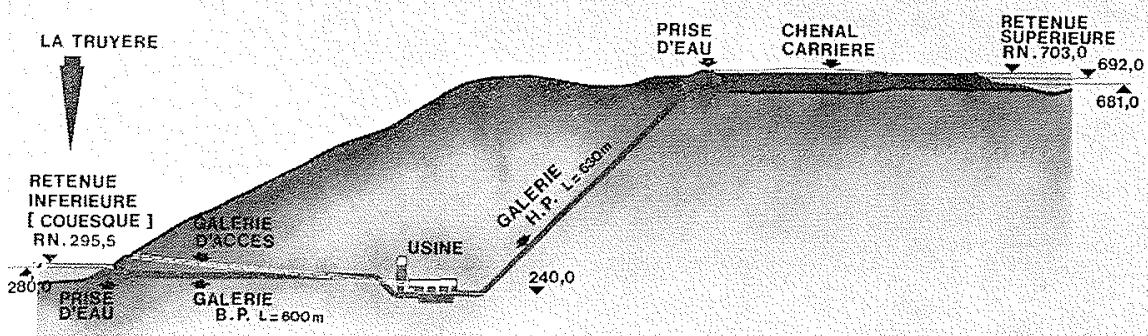

$0.00 \mathrm{~m} \quad \mathrm{~S}^{500 \mathrm{~m}}$ 

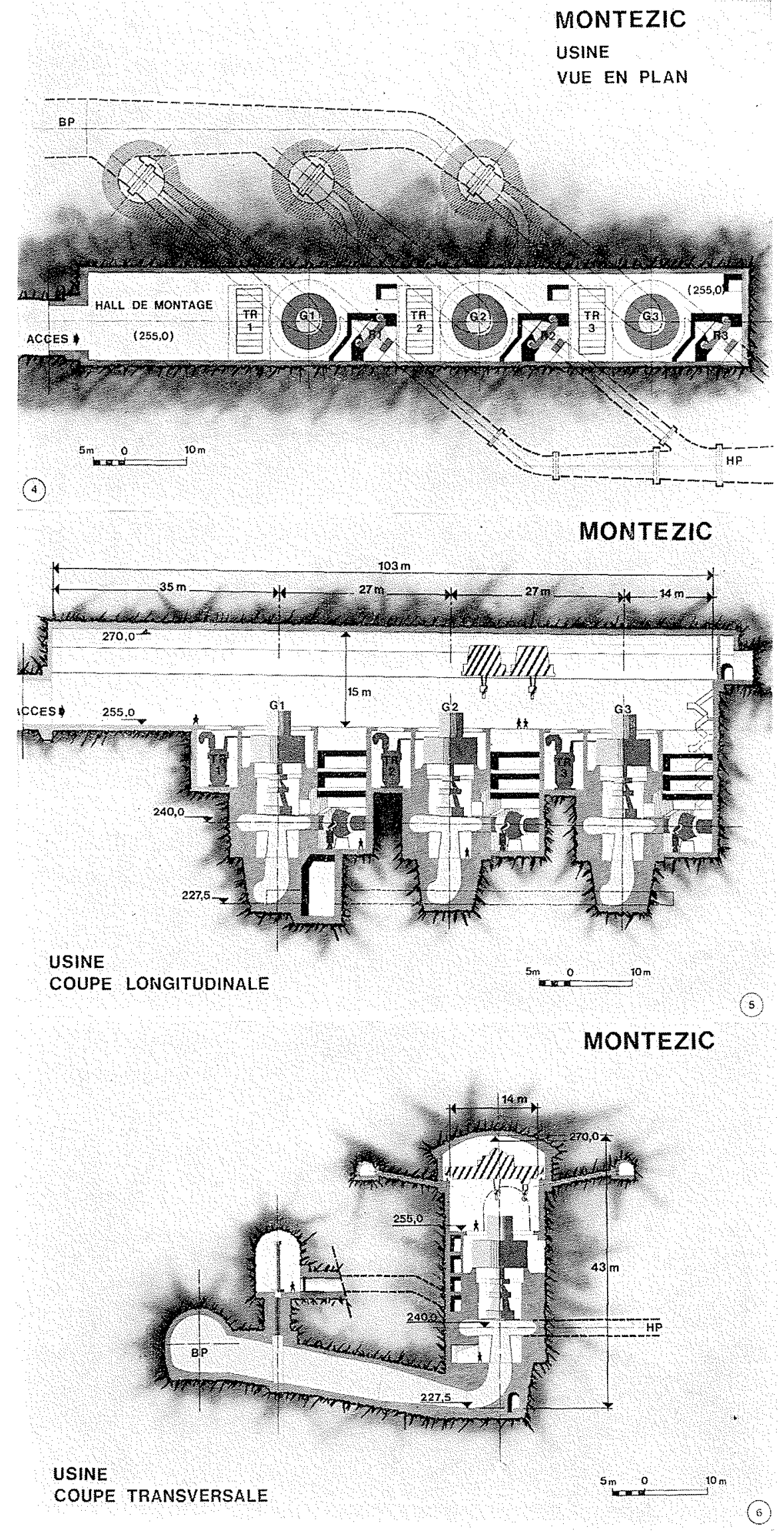
Les diamètres, longueurs et pressions mises en jeu conduiraient à une dépense fort importante. Afin d'aborder l'étude de ce problème, deux sondages verticaux d'une profondeur de $150 \mathrm{~m}$ ont été réalisés durant la campagne de reconnaissances géologiques de l'été 1971, l'un implanté dans le granite, l'autre dans le schiste. Chacun d'eux a été l'objet d'essais d'eau sous une pression de 30 bars.

Le sondage réalisé dans les schistes n'a pas donné de résultats satisfaisants quant à son étanchéité. Par contre, celui implanté dans les granites a montré que dès que la zone d'altération était dépassée, les pertes d'eau étaient pratiquement nulles sur toute la hauteur du forage. Compte tenu de ces premiers résultats encourageants, cette étude va être poursuivie l'été prochain par la mise en pression d'un tronçon de galerie de $2,50 \mathrm{~m}$ de diamètre et de $150 \mathrm{~m}$ de long. Il sera soumis à des cycles atteignant les pressions maximales de 20 - 40 et 60 bars. Quoi qu'il en soit, les résultats déjà obtenus invitent à placer les ouvrages haute pression dans les granites comme d'ailleurs l'usine, pour des considérations liées à son exécution.

Le schéma figuré sur le profil en long des installations est un schéma possible. L'usine, telle qu'elle est implantée, devrait se trouver dans les granites. On pense même, ce qui est souhaitable, qu'il sera possible de la rapprocher notablement de la retenue inférieure. Cette indétermination sera levée quand aura été exécutée une galerie de reconnaissance qui déterminera, au niveau de l'usine, la position du contact entre les schistes et les granites. Le début d'exécution de cette galerie est proche et c'est à son extrémité aval que seront réalisés les essais de mise en pression dont il vient d'être question.

Sur ce schéma, la galerie haute pression est inclinée. Sa longueur est de $630 \mathrm{~m}$, les $100 \mathrm{~m}$ précédant l'usine recevant un blindage. A l'amont, un revêtement en béton est seulement prévu. Le but de ce revêtement n'est pas d'assurer l'étanchéité, car il a été montré, lors des essais exécutés à Revin notamment, que sous les pressions mises en jeu, le béton se fissurait; son but est seulement d'améliorer le coefficient de rugosité et d'éviter que ne se détachent des parois des morceaux de rocher. La section de cette galerie est de $40 \mathrm{~m}^{2}$, son diamètre de $7 \mathrm{~m}$. Compte tenu de sa longueur, les régimes transitoires ne posent aucun problème. Une solution comportant un puits vertical et une galerie horizontale n'est pas exclue. En tête, la prise d'eau est munie d'une grille et d'une vanne.

L'adduction basse pression comporte une galerie unique de $600 \mathrm{~m}$ de longueur et de section $67 \mathrm{~m}^{2}$. Sa longueur rend nécessaire une cheminée d'équilibre aval qui pourrait être supprimée s'il était possible de rapprocher l'usine de la retenue inférieure.

Cette adduction peut être isolée de la retenue par une vanne placée à la prise d'eau inférieure.

\section{L'usine (fig. 4.5 et 6 )}

Rien n'a encore été dit à propos des puissances mises en jeu aussi bien pour les pompes que pour les turbines.

Pour assurer le cycle de fonctionnement hebdomadaire parfait et une utilisation en turbine de $12 \mathrm{~h}$, la capacité nécessaire est six fois la capacité nécessitée par un fonctionnement selon un cycle journalier. Celle-ci serait donc de $30 / 6 \mathrm{Mm}^{3}=5 \mathrm{Mm}^{3}$ qui seraient à élever du bassin inférieur vers le bassin supérieur durant les $8 \mathrm{~h}$ creuses de chaque jour ouvrable. Le débit moyen de l'installation, en pompage, sera de $174 \mathrm{~m}^{3} / \mathrm{s}$.

En fonction de la variation de la hauteur de chute, il pourra varier, en fait, au cours du cycle de : 160 à $190 \mathrm{~m}^{3} / \mathrm{s}$.

Pour le fonctionnement en turbine, on dispose d'un volume de $5+25 / 5=10 \mathrm{Mm}^{3}$ à utiliser pendant $12 \mathrm{~h}$, ce qui conduit à un débit moyen de $232 \mathrm{~m}^{3} / \mathrm{s}$ qui pourra varier, au cours du cycle, entre 220 et $240 \mathrm{~m}^{3} / \mathrm{s}$.

Ces débits associés aux hauteurs de chutes nettes correspondantes donnent les variations de puissance suivantes:

- en utilisation en turbine, de $863 \mathrm{MW}$ à $732 \mathrm{MW}$;

- en utilisation en pompe, de $756 \mathrm{MW}$ à $810 \mathrm{MW}$.

Les puissances installées sont donc de :

- $863 \mathrm{MW}$ pour les turbines et

- $810 \mathrm{MW}$ pour les pompes.

La puissance moyenne maximale serait de l'ordre de $790 \mathrm{MW}$. Ce qui est compatible avec les besoins du réseau français vers les années 1980 , c'est-à-dire à peu près au moment de la mise en service de l'installation.

Le projet d'usine présenté ici comporte trois groupes verticaux de $287 \mathrm{MW}$. Leur vitesse de rotation est de $375 \mathrm{tr} / \mathrm{mn}$. Le diamètre de la roue $4,52 \mathrm{~m}$.

Une solution avec deux groupes seulement, de puissance unitaire $430 \mathrm{MW}$, est à l'étude.

La hauteur de chute peu supérieure à $400 \mathrm{~m}$ autorise le choix de groupes Francis réversibles. Ce type de matériel, principalement lorsqu'il s'agit d'une usine souterraine, amène des simplifications notables dans la conception du génie civil de l'usine.

La contre-pression à maintenir sur la zone nécessite le calage de son axe à $40 \mathrm{~m}$ au-dessous du plan d'eau minimum dans le bassin inférieur, c'est-à-dire à la cote 240 . Cet impératif serait une gêne s'il s'agissait d'une usine à l'air libre.

Le grand axe de l'excavation de l'usine est disposé parallèlement à la direction des adductions. Les groupes sont raccordés à l'adduction haute pression par un collecteur dont le blindage se poursuit une centaine de mètres à l'amont de l'usine. Les aspirateurs sont «piqués» sur la galerie basse pression.

La galerie d'accès, longue de $550 \mathrm{~m}$, dessert la plage de montage, située en bout d'usine, au niveau de l'arase supérieure des alternateurs.

A chaque groupe est associé un robinet sphérique de pied et un transformateur.

L'angle d'arrivée de la tubulure d'alimentation sur le long pan de l'usine, fixé à $45^{\circ}$, conduit à la largeur minimale de l'excavation. Cette largeur est d'une quinzaine de mètres. Sa longueur est de $103 \mathrm{~m}$ et la hauteur depuis le fond de l'aspirateur jusqu'à la clé de voûte de $43 \mathrm{~m}$.

Chaque groupe peut être isolé des ouvrages basse pression par une vanne batardeau.

Le mode de lancement des groupes en pompes n'est pas encore déterminé. Il est regrettable qu'il ne soit pas possible, pour faire ce choix, de bénéficier de l'expérience de Revin où l'un des quatre groupes est prévu à démarrage direct sur le réseau.

L'énergie sera livrée ou délivrée sous la tension $380 \mathrm{kV}$ au poste de Rueyres, situé tout à côté de l'usine de Brommat d'où sera probablement télécommandée l'usine de Montezic. 
Discussion

Président : M. H. DE Maublanc

M. de Maublanc remercie M. Morenon pour la clarté avec laquelle il a décrit le beau projet de Montezic.

Il remarque que les puissances en jeu, tant à Revin qu'à Montezic, sont de l'ordre de grandeur de celles des unités thermiques les plus puissantes d'aujourd'hui $(700 \mathrm{MW})$ et un peu inférieures à celles des centrales nucléaires récentes (Bugey, Saint-Laurent ef bientôt Fessenheim). La communication de M. Gérard nous a montré les raisons de ce fait.

Sur une question de M. De Maublanc, M. Morenon précise que les investissements sont de l'ordre de 35 milliards d'anciens francs pour laménagement de Montezic et de plusieurs milliards pour les ouvrages de transport de l'énergie.

M. LE Granché intervient dans les termes suivants:

$\mathrm{Ne}$ possédant pas les caractéristiques de votre roue d'essai, il me paraît difficile de pouvoir effectuer une étude complète des régimes transitoires qui me semble nécessaire, compte tenu des longueurs respectives des adductions amont et aval; il me paraît utile de préciser que dans le cas des centrales équipées de turbinespompes, l'implantation de la centrale doit être faite après l'étude complète des régimes transitores, le critère de perméabilité du terrain obligeant simplement à blinder ou non les adductions.

M. MORENON répond que le problème ne peut être résolu que par approximations successives car bien des facteurs entrent en jeu dans le choix ci-dessus.

Dans des cas analogues, nous serons peut-être amenés dans l'avenir, observe M. le Président, ì nous pencher par priorité sur les problèmes hydrauliques et la spécification détaillée des groupes hydroélectro-mécaniques pour finalement dimensionner au mieux l'ensemble de laménagement. Cela conduirait à inverser en quelque sorte l'ordre des études traditionnellement adopté dans le cas d'une usine hydroélectrique classique; la question mérite réflexion et sera examinée plus en détail au cours des séances suivantes.

Personne ne demandant la parole, M. le Président lève la séance à $12 \mathrm{~h} 15$, en remerciant ceux qui ont contribué à l'animer. 